\title{
Radial Symmetry and Monotonicity of Solutions to a System Involving Fractional p-Laplacian in a Ball
}

\author{
Linfen Cao (D), Xiaoshan Wang, and Zhaohui Dai \\ Henan Engineering Laboratory for Big Data Statistical Analysis and Optimal Control, \\ College of Mathematics and Information Science, Henan Normal University, Xinxiang 453007, China
}

Correspondence should be addressed to Linfen Cao; linfencao6@163.com

Received 24 April 2018; Accepted 26 June 2018; Published 1 August 2018

Academic Editor: Pietro d'Avenia

Copyright (C) 2018 Linfen Cao et al. This is an open access article distributed under the Creative Commons Attribution License, which permits unrestricted use, distribution, and reproduction in any medium, provided the original work is properly cited.

In this paper, we study a nonlinear system involving the fractional p-Laplacian in a unit ball and establish the radial symmetry and monotonicity of its positive solutions. By using the direct method of moving planes, we prove the following result. For $0<s, \mathrm{t}<$ $1, \mathrm{p}>0$, if $u$ and $v$ satisfy the following nonlinear system $\left\{(-\Delta)_{p}^{s} u(x)=f(v(x)) ;(-\Delta)_{p}^{t} v(x)=g(u(x)), x \in B_{1}(0) ; u(x), v(x)=\right.$ $0, x \notin B_{1}(0)$. $\}$ and $f, g$ are nonnegative continuous functions satisfying the following: (i) $f(r)$ and $g(r)$ are increasing for $r>0$; (ii) $f^{\prime}(r) / r^{p-2}, g^{\prime}(r) / r^{p-2}$ are bounded near $r=0$. Then the positive solutions $(u, v)$ must be radially symmetric and monotone decreasing about the origin.

\section{Introduction}

Nonlinear equations involving fractional powers of the Laplacian are currently actively studied. The fractional and nonlocal operators of elliptic type due to concrete real world have been applied in finance, thin obstacle problem, optimization, quasi-geostrophic flow, etc. In recent years, there have been tremendous interests in developing the problems related to fractional Laplacian (see in [1-5] and the reference therein). Fractional p-Laplacian is a generalization of the fractional Laplacian, but there are few interesting results about these nonlinear equations involving fractional p-Laplacian. In [6], the authors considered the positive solution to the following problem involving the fractional p-Laplacian:

$$
(-\Delta)_{p}^{s} u(x)=g(u(x)), \quad x \in \mathbb{R}^{n} .
$$

where $(-\Delta)_{p}^{s}$ is defined by

$$
\begin{aligned}
& (-\Delta)_{p}^{s} u(x) \\
& \quad=C_{n, s p} P V \int_{\mathbb{R}^{n}} \frac{|u(x)-u(z)|^{p-2}[u(x)-u(z)]}{|x-z|^{n+s p}} d z,
\end{aligned}
$$

where $P V$ stands for the Cauchy principal value. In order to make sense the integral, we require that $u \in L_{s p} \cap C_{l o c}^{1,1}$ with

$$
L_{s p}=\left\{u \in L_{l o c}^{1} \mid \int_{R^{n}} \frac{|u(x)|^{p-1}}{1+|x|^{n+s p}} d x<\infty\right\} .
$$

They show that if $u(x) \in L_{s p} \cap C_{l o c}^{1,1}$ is a positive solution to (1) with $\lim _{|x| \rightarrow \infty} u(x)=0$, and $g^{\prime}(s) \leq 0$ for $s>0$ sufficiently small, then $u$ must be radially symmetric and monotone decreasing about some point in $\mathbb{R}^{n}$.

The fractional p-Laplacian $(-\Delta)_{p}^{s}$ is a special case of the following fully nonlinear operators $F_{\alpha}$ :

$$
F_{\alpha}(u(x))=C_{n, \alpha} P V \int_{\mathbb{R}^{n}} \frac{G(u(x)-u(y))}{|x-y|^{n+\alpha}} d y,
$$

where $G$ is at least local Lipschitz continuous, $G(0)=0$, and $\alpha>0$ (see [7] for the introductions of these operators, one also see [8] for systems case). In this case $\alpha=s p$ and $G(t)=$ $|t|^{p-2} t$.

When $p=2$, the fractional p-Laplacian $(-\Delta)_{p}^{s}$ becomes the well-known fractional Laplacian operator $(-\Delta)^{s}$. In [2], 
the authors considered the following system involving fractional Laplacian on the whole space:

$$
\begin{aligned}
& (-\Delta)^{s} u(x)=f(v(x)), \\
& (-\Delta)^{t} v(x)=g(u(x)), \quad x \in \mathbb{R}^{n}, \\
& u(x), v(x) \geq 0, \quad x \in \mathbb{R}^{n} .
\end{aligned}
$$

Assume that, for $r \geq 0, f, g$ are nonnegative continuous functions satisfying the following: (a) $f(r)$ and $g(r)$ are nondecreasing about $r$; (b) $f(r) / r^{p}, g(r) / r^{q}$ are bounded near $r=0$ and nonincreasing with $p=(n+2 s) /(n-2 t)$ and $q=(n+2 t) /(n-2 s)$. If $u$ and $v$ are nonnegative solutions for (5), then either $u$ and $v$ are constant or $f(v)=C_{1} v^{(n+2 s) /(n-2 t)}$ and $g(u)=C_{2} u^{(n+2 t) /(n-2 s)}$.

Motivated by the ideas of $[2,6]$, our main concern in this paper is to study the following nonlinear system involving fractional p-Laplacian in a unit ball:

$$
\begin{aligned}
& (-\Delta)_{p}^{s} u(x)=f(v(x)), \\
& (-\Delta)_{p}^{t} v(x)=g(u(x)), \quad x \in B_{1}(0), \\
& u(x), v(x)=0, \quad x \notin B_{1}(0),
\end{aligned}
$$

where $0<s, t<1$. We obtain the following main theorem by the direct method of moving planes.

Theorem 1. Assume that $u(x) \in L_{s p}\left(B_{1}(0)\right) \cap C_{l o c}^{1,1}\left(B_{1}(0)\right)$ and $v(x) \in L_{t p}\left(B_{1}(0)\right) \cap C_{\text {loc }}^{1,1}\left(B_{1}(0)\right)$ are positive solutions of system (6); $f, g$ are nonnegative continuous functions satisfying the following:

(i) $f(r)$ and $g(r)$ are increasing about $r>0$.

(ii) $f^{\prime}(r) / r^{p-2}, g^{\prime}(r) / r^{p-2}$ are bounded near $r=0$.

Then the positive solutions $(u, v)$ must be radially symmetric and monotone decreasing about the origin.

Remark 2. In $[3,9]$, the authors introduced the direct method of moving planes, respectively, to prove symmetry, monotonicity, and nonexistence of solutions to various differential equations on the whole space and on a half space, etc., such as ones involving fractional Laplacian in $[2,3]$, fractional pLaplacian in [6], and fully nonlinear operators $F_{\alpha}$ in $[4,8]$. This direct method of moving planes has some advantages, which overcome the necessary of imposing extra assumption on the solutions when using the extension method (see also [10]) or the equivalent integral equation method (see also [11]).

The key ingredients of the direct method are maximum principle for antisymmetric functions and key boundary estimate lemma. For convenience we put both of them in the appendix.

\section{Proof of Theorem 1}

As usual, we choose any direction to the $x_{1}$ direction; let

$$
T_{\lambda}=\left\{x=\left(x_{1}, x_{2}, \ldots, x_{n}\right) \in \mathbb{R}^{n} \mid x_{1}=\lambda, \lambda \in \mathbb{R}\right\}
$$

be the moving planes,

$$
\Sigma_{\lambda}=\left\{x \in \mathbb{R}^{n} \mid x_{1}<\lambda\right\}
$$

be the region to the left of the plane, and

$$
x^{\lambda}=\left(2 \lambda-x_{1}, x_{2}, \ldots, x_{n}\right)
$$

be the reflection of $x$ about plane $T_{\lambda}$, and $u_{\lambda}(x)=u\left(x^{\lambda}\right)$, $v_{\lambda}(x)=v\left(x^{\lambda}\right)$.

Let

$$
\begin{aligned}
& U_{\lambda}(x)=u_{\lambda}(x)-u(x), \\
& V_{\lambda}(x)=v_{\lambda}(x)-v(x)
\end{aligned}
$$

denote

$$
\Omega_{\lambda}=\Sigma_{\lambda} \cap B_{1}(0) .
$$

Then in $\Omega_{\lambda}$, we have

$$
\begin{aligned}
& (-\Delta)_{p}^{s} u_{\lambda}(x)-(-\Delta)_{p}^{s} u(x)=f\left(v_{\lambda}(x)\right)-f(v(x)) \\
& =f^{\prime}\left(\xi_{\lambda}(x)\right) V_{\lambda}(x)
\end{aligned}
$$

and

$$
\begin{aligned}
& (-\Delta)_{p}^{t} v_{\lambda}(x)-(-\Delta)_{p}^{t} v(x)=g\left(u_{\lambda}(x)\right)-g(u(x)) \\
& =g^{\prime}\left(\eta_{\lambda}(x)\right) U_{\lambda}(x)
\end{aligned}
$$

where $\xi_{\lambda}(x)$ is valued between $v_{\lambda}(x)$ and $v(x) ; \eta_{\lambda}(x)$ is valued between $u_{\lambda}(x)$ and $u(x)$.

Step 1. In this step, we show that, for $\lambda>-1$ and sufficiently closed to -1 , it holds

$$
U_{\lambda}(x), V_{\lambda}(x) \geq 0, \quad \forall x \in \Omega_{\lambda} .
$$

We prove (14) by a contradiction argument, then there is at least one inequality that does not hold. Without loss of generality, we assume $U_{\lambda}(x)<0$ at some point in $\Omega_{\lambda}$, then there exists some $\tilde{x} \in \Omega_{\lambda}$, such that

$$
U_{\lambda}(\tilde{x})=\min _{\Omega_{\lambda}} U_{\lambda}(x)<0,
$$

then we will show that $V_{\lambda}(\tilde{x})<0$ and derive a contradiction.

Since the fractional p-Laplacian is a special case of the fully nonlinear operators $F_{\alpha}($.$) , when$

$$
\begin{aligned}
\alpha & =s p, \\
G(t) & =|t|^{p-2} t,
\end{aligned}
$$

we need an analysis lemma about $G(t)$ in [6].

Lemma 3. For $G(t)=|t|^{p-2} t$, it is well-known that, by the mean value theorem, we have

$$
G\left(t_{2}\right)-G\left(t_{1}\right)=G^{\prime}(\xi)\left(t_{2}-t_{1}\right) .
$$

Then there exists a constant $c_{0}>0$, such that

$$
|\xi| \geq c_{0} \max \left\{\left|t_{1}\right|,\left|t_{2}\right|\right\} \text {. }
$$

Then we have 


$$
\begin{aligned}
& (-\Delta)_{p}^{s} u_{\lambda}(\widetilde{x})-(-\Delta)_{p}^{s} u(\widetilde{x})=C_{n, s p} P V \int_{\mathbb{R}^{n}} \frac{G\left(u_{\lambda}(\tilde{x})-u_{\lambda}(y)\right)-G(u(\widetilde{x})-u(y))}{|\widetilde{x}-y|^{n+s p}} d y \\
& =C_{n, s p} P V \int_{\Sigma_{\lambda}} \frac{G\left(u_{\lambda}(\tilde{x})-u_{\lambda}(y)\right)-G(u(\tilde{x})-u(y))}{|\tilde{x}-y|^{n+s p}} d y+C_{n, s p} P V \int_{\Sigma_{\lambda}} \frac{G\left(u_{\lambda}(\tilde{x})-u(y)\right)-G\left(u(\tilde{x})-u_{\lambda}(y)\right)}{\left|\tilde{x}-y^{\lambda}\right|^{n+s p}} d y \\
& =C_{n, s p} P V\left\{\int_{\Sigma_{\lambda}}\left[G\left(u_{\lambda}(\widetilde{x})-u_{\lambda}(y)\right)-G(u(\widetilde{x})-u(y))\right]\left(\frac{1}{|\widetilde{x}-y|^{n+s p}}-\frac{1}{\left|\widetilde{x}-y^{\lambda}\right|^{n+s p}}\right) d y\right. \\
& \left.+\int_{\Sigma_{\lambda}} \frac{G\left(u_{\lambda}(\tilde{x})-u_{\lambda}(y)\right)-G(u(\tilde{x})-u(y))+G\left(u_{\lambda}(\tilde{x})-u(y)\right)-G\left(u(\tilde{x})-u_{\lambda}(y)\right)}{\left|\widetilde{x}-y^{\lambda}\right|^{n+s p}} d y\right\} \\
& \leq C_{n, s p} P V \int_{\Sigma_{\lambda}} \frac{G\left(t_{1}(\tilde{x}, y)\right)-G\left(t_{2}(\tilde{x}, y)\right)+G\left(t_{3}(\tilde{x}, y)\right)-G\left(t_{4}(\tilde{x}, y)\right)}{\left|\tilde{x}-y^{\lambda}\right|^{n+s p}} d y \leq C_{n, s p} P V U_{\lambda}(\tilde{x}) \\
& \cdot \int_{\Sigma_{\lambda}} \frac{G^{\prime}(\xi(y))+G^{\prime}(\eta(y))}{\left|\widetilde{x}-y^{\lambda}\right|^{n+s p}} d y=C_{n, s p} P V U_{\lambda}(\widetilde{x}) I(\widetilde{x}) .
\end{aligned}
$$

Here

$$
\begin{aligned}
& t_{1}(\tilde{x}, y)=u_{\lambda}(\tilde{x})-u_{\lambda}(y), \\
& t_{2}(\tilde{x}, y)=u(\tilde{x})-u_{\lambda}(y), \\
& t_{3}(\tilde{x}, y)=u_{\lambda}(\tilde{x})-u(y), \\
& t_{4}(\tilde{x}, y)=u(\tilde{x})-u(y),
\end{aligned}
$$

and

$$
\begin{aligned}
& t_{1}(\tilde{x}, y)<\xi(\tilde{x}, y)<t_{2}(\tilde{x}, y), \\
& t_{3}(\tilde{x}, y)<\eta(\tilde{x}, y)<t_{4}(\tilde{x}, y) .
\end{aligned}
$$

We now estimate $I(\tilde{x})$ for $\tilde{x} \in \Omega_{\lambda}$ to derive a contradiction when $\lambda$ is sufficiently close to -1 . To this end, we need to use Lemma 3. Let $D=\Sigma_{\lambda} \backslash \Omega_{\lambda}$. Noticing that $u(y)=0$ in $D$, for some positive constant $c_{1}$ and $c_{2}$, we have

$$
\begin{aligned}
I(\tilde{x}) & =\int_{\Sigma_{\lambda}} \frac{(p-2)\left(|\xi|^{p-2}+|\eta|^{p-2}\right)}{\left|\tilde{x}-y^{\lambda}\right|^{n+s p}} d y \\
& \geq \int_{\Sigma_{\lambda}} \frac{(p-2) c_{0}\left(\left|t_{2}\right|^{p-2}+\left|t_{4}\right|^{p-2}\right)}{\left|\tilde{x}-y^{\lambda}\right|^{n+s p}} d y \\
& \geq \int_{\Sigma_{\lambda}} \frac{c_{1}\left|t_{4}(y)\right|^{p-2}}{\left|\tilde{x}-y^{\lambda}\right|^{n+s p}} d y \geq c_{1} \int_{D} \frac{u^{p-2}(\tilde{x})}{\left|\widetilde{x}-y^{\lambda}\right|^{n+s p}} d y \\
& \geq \frac{c_{2} u^{p-2}(\tilde{x})}{\delta^{s p}},
\end{aligned}
$$

where $\delta=\lambda+1$ is the width of the region $\Omega_{\lambda}$ in the $x_{1}$-direction.

Hence

$$
(-\Delta)_{p}^{s} u_{\lambda}(\tilde{x})-(-\Delta)_{p}^{s} u(\tilde{x}) \leq C U_{\lambda}(\tilde{x}) \frac{u^{p-2}(\tilde{x})}{\delta^{s p}}<0 .
$$

Together with $(12)$ and $f^{\prime}(\cdot)>0$, it is easy to deduce that

$$
V_{\lambda}(\tilde{x})<0
$$

and

$$
C U_{\lambda}(\tilde{x}) \frac{u^{p-2}(\tilde{x})}{\delta^{s p}} \geq f^{\prime}\left(\xi_{\lambda}(\tilde{x})\right) V_{\lambda}(\tilde{x}),
$$

and we have

$$
U_{\lambda}(\tilde{x}) \geq \frac{C f^{\prime}\left(\xi_{\lambda}(\tilde{x})\right) V_{\lambda}(\tilde{x}) \delta^{s p}}{u^{p-2}(\tilde{x})} .
$$

From (24), there exists $\bar{x} \in \Omega_{\lambda}$ such that

$$
V_{\lambda}(\bar{x})=\min _{\Omega_{\lambda}} V_{\lambda}(x)<0 .
$$

Similar to (23), we can derive

$$
(-\Delta)_{p}^{t} v_{\lambda}(\bar{x})-(-\Delta)_{p}^{t} v(\bar{x}) \leq C V_{\lambda}(\bar{x}) \frac{v^{p-2}(\bar{x})}{\delta^{t p}}<0,
$$

and, combining (13) and (26), we can deduce that

$$
\begin{aligned}
C V_{\lambda}(\bar{x}) \frac{v^{p-2}(\bar{x})}{\delta^{t p}} \geq g^{\prime}\left(\eta_{\lambda}(\bar{x})\right) U_{\lambda}(\bar{x}) \\
\geq g^{\prime}\left(\eta_{\lambda}(\bar{x})\right) U_{\lambda}(\tilde{x}) \\
\geq \frac{C f^{\prime}\left(\xi_{\lambda}(\tilde{x})\right) V_{\lambda}(\tilde{x}) \delta^{s p} g^{\prime}\left(\eta_{\lambda}(\bar{x})\right)}{u^{p-2}(\tilde{x})},
\end{aligned}
$$

then

$$
\begin{aligned}
V_{\lambda}(\bar{x}) & \geq \frac{f^{\prime}\left(\xi_{\lambda}(\tilde{x})\right) g^{\prime}\left(\eta_{\lambda}(\bar{x})\right)}{u^{p-2}(\tilde{x}) v^{p-2}(\bar{x})} \delta^{(s+t) p} V_{\lambda}(\tilde{x}) \\
& =A V_{\lambda}(\tilde{x}),
\end{aligned}
$$


where

$$
A=\frac{f^{\prime}\left(\xi_{\lambda}(\widetilde{x})\right) g^{\prime}\left(\eta_{\lambda}(\bar{x})\right)}{u^{p-2}(\widetilde{x}) v^{p-2}(\bar{x})} \delta^{(s+t) p} .
$$

When $\lambda$ is sufficiently close to $-1, \delta$ becomes sufficiently small; combining assumptions (i) and (ii) in Theorem 1, we can deduce that $A$ is sufficiently small and we can let $A<1$. According to (24), inequality (30) becomes

$$
V_{\lambda}(\bar{x}) \geq A V_{\lambda}(\tilde{x})>V_{\lambda}(\tilde{x}) .
$$

This is a contradiction with the fact that $\bar{x}$ is the negative minimum of $V_{\lambda}(x)$. Therefore (14) must be true for $\lambda$ sufficiently closed to -1 .

Step 2. Inequality (14) provides a starting point, from which we move plane $T_{\lambda}$ toward the right as long as (14) holds to its limiting position to show that $u, v$ are symmetric about the limiting plane. More precisely, let

$$
\lambda_{0}=\left\{\lambda \leq 0 \mid U_{\mu}(x), V_{\mu}(x) \geq 0, x \in \Omega_{\mu}, \forall \mu \leq \lambda\right\} .
$$

We will show that

$$
\lambda_{0}=0,
$$

and

$$
\begin{aligned}
& U_{\lambda_{0}}(x) \equiv 0, \\
& V_{\lambda_{0}}(x) \equiv 0,
\end{aligned}
$$

$$
x \in \Omega_{\lambda_{0}} .
$$

Suppose that $\lambda_{0}<0$, and $U_{\lambda_{0}}(x), V_{\lambda_{0}}(x) \geq 0$ but $U_{\lambda_{0}}(x) \not \equiv$ 0 and $V_{\lambda_{0}}(x) \neq 0$, from that nonnegative functions $U_{\lambda_{0}}(x)$ or $V_{\lambda_{0}}(x)$ are positive at some point in $\Omega_{\lambda_{0}}$; combining (12), (13), and the fact that $f^{\prime}(\cdot), g^{\prime}(\cdot)>0$, by using the strong maximum principle of Theorem A.1, we obtain

$$
\begin{aligned}
& U_{\lambda_{0}}(x)>0, \\
& V_{\lambda_{0}}(x)>0, \\
& \quad x \in \Omega_{\lambda_{0}} .
\end{aligned}
$$

In fact, if there exists some $x^{0} \in \Omega_{\lambda_{0}}$, such that

$$
U_{\lambda_{0}}\left(x^{0}\right)=\min _{\Omega_{\lambda_{0}}} U_{\lambda_{0}}=0,
$$

we have,

$$
\begin{aligned}
& (-\Delta)_{p}^{s} u_{\lambda_{0}}\left(x^{0}\right)-(-\Delta)_{p}^{s} u\left(x^{0}\right)=C_{n, s p} P V\left\{\int _ { \Sigma _ { \lambda _ { 0 } } } [ G ( u _ { \lambda _ { 0 } } ( x ^ { 0 } ) - u _ { \lambda _ { 0 } } ( y ) ) - G ( u ( x ^ { 0 } ) - u ( y ) ) ] \left(\frac{1}{\left|x^{0}-y\right|^{n+s p}}\right.\right. \\
& \left.-\frac{1}{\left|x^{0}-y^{\lambda_{0}}\right|^{n+s p}}\right) d y \\
& \left.+\int_{\Sigma_{\lambda_{0}}} \frac{G\left(u_{\lambda_{0}}\left(x^{0}\right)-u_{\lambda_{0}}(y)\right)-G\left(u\left(x^{0}\right)-u(y)\right)+G\left(u_{\lambda_{0}}\left(x^{0}\right)-u(y)\right)-G\left(u\left(x^{0}\right)-u_{\lambda_{0}}(y)\right)}{\left|x^{0}-y^{\lambda_{0}}\right|^{n+s p}} d y\right\} \\
& =C_{n, s p} P V\left\{\int_{\Sigma_{\lambda_{0}}}\left[G\left(u_{\lambda_{0}}\left(x^{0}\right)-u_{\lambda_{0}}(y)\right)-G\left(u\left(x^{0}\right)-u(y)\right)\right]\left(\frac{1}{\left|x^{0}-y\right|^{n+s p}}-\frac{1}{\left|x^{0}-y^{\lambda_{0}}\right|^{n+s p}}\right) d y+U_{\lambda_{0}}\left(x^{0}\right)\right. \\
& \left.\cdot \int_{\Sigma_{\lambda_{0}}} \frac{G^{\prime}(\xi(y))+G^{\prime}(\eta(y))}{\left|x^{0}-y^{\lambda_{0}}\right|^{n+s p}} d y\right\}=C_{n, s p} P V\left\{\int _ { \Sigma _ { \lambda _ { 0 } } } [ G ( u _ { \lambda _ { 0 } } ( x ^ { 0 } ) - u _ { \lambda _ { 0 } } ( y ) ) - G ( u ( x ^ { 0 } ) - u ( y ) ) ] \left(\frac{1}{\left|x^{0}-y\right|^{n+s p}}\right.\right. \\
& \left.-\frac{1}{\left|x^{0}-y^{\lambda_{0}}\right|^{n+s p}}\right) d y<0 .
\end{aligned}
$$

On the other hand,

$$
\begin{aligned}
& (-\Delta)_{p}^{s} u_{\lambda_{0}}\left(x^{0}\right)-(-\Delta)_{p}^{s} u\left(x^{0}\right) \\
& \quad=f\left(v_{\lambda_{0}}(x)\right)-f(v(x))=f^{\prime}\left(\xi_{\lambda_{0}}(x)\right) V_{\lambda_{0}}(x) \\
& \geq 0 .
\end{aligned}
$$

A contradiction with (38), hence (36) must be true.
By the definition of $\lambda_{0}$, there exists a sequence $0>\lambda_{k} \searrow$ $\lambda_{0}$, and $x^{k} \in \Omega_{\lambda_{k}}$, such that

$$
U_{\lambda_{k}}\left(x^{k}\right)=\min _{\Sigma_{\lambda_{k}}} U_{\lambda_{k}}(x)<0, \quad \nabla U_{\lambda_{k}}\left(x^{k}\right)=0,
$$

and, combining (23) and (24), there also exists a sequence $0>$ $\lambda_{k} \searrow \lambda_{0}$ and $x^{l} \in \Omega_{\lambda_{k}}$, such that

$$
V_{\lambda_{k}}\left(x^{l}\right)=\min _{\Sigma_{\lambda_{k}}} V_{\lambda_{k}}(x)<0, \quad \nabla V_{\lambda_{k}}\left(x^{l}\right)=0 .
$$


There is a subsequence of $\left\{x^{k}\right\}$ and $\left\{x^{l}\right\}$ that converges to some point $x^{0}$ and $x^{1}$, respectively. And from (40), (41), and the continuity of $U_{\lambda}(x), V_{\lambda}(x)$ and its derivative with respect to both $x$ and $\lambda$, we have

$$
U_{\lambda_{0}}\left(x^{0}\right) \leq 0, \text { hence } x^{o} \in \partial \Sigma_{\lambda_{0}} ; \nabla U_{\lambda_{0}}\left(x^{0}\right)=0,
$$

and

$$
V_{\lambda_{0}}\left(x^{1}\right) \leq 0, \text { hence } x^{1} \in \partial \Sigma_{\lambda_{0}} ; \nabla V_{\lambda_{0}}\left(x^{1}\right)=0 .
$$

Then, we have

$$
\begin{aligned}
\varlimsup_{\delta_{k} \longrightarrow 0} \frac{1}{\delta_{k}}\left\{(-\Delta)_{p}^{s} u_{\lambda_{k}}\left(x^{k}\right)-(-\Delta)_{p}^{s} u\left(x^{k}\right)\right\} \\
=\frac{f^{\prime}\left(\xi_{\lambda_{k}}\left(x^{k}\right)\right) V_{\lambda_{k}}\left(x^{k}\right)}{\delta_{k}} \\
\geq \frac{f^{\prime}\left(\xi_{\lambda_{k}}\left(x^{k}\right)\right) V_{\lambda_{k}}\left(x^{l}\right)}{\delta_{k}} \longrightarrow 0,
\end{aligned}
$$

and

$$
\begin{aligned}
\varlimsup_{\delta_{k}} \rightarrow 0 & \frac{1}{\delta_{k}}\left\{(-\Delta)_{p}^{t} v_{\lambda_{k}}\left(x^{l}\right)-(-\Delta)_{p}^{t} v\left(x^{l}\right)\right\} \\
= & \frac{g^{\prime}\left(\eta_{\lambda_{k}}\left(x^{l}\right)\right) U_{\lambda_{k}}\left(x^{l}\right)}{\delta_{k}} \geq \frac{g^{\prime}\left(\eta_{\lambda_{k}}\left(x^{l}\right)\right) U_{\lambda_{k}}\left(x^{k}\right)}{\delta_{k}} \\
& \longrightarrow 0 .
\end{aligned}
$$

This will contradict with Theorem A.2. Therefore, we have

$$
\begin{aligned}
\lambda_{0} & =0, \\
U_{\lambda_{0}}(x) & =V_{\lambda_{0}}(x) \equiv 0, \\
& \forall x \in \Omega_{\lambda_{0}} .
\end{aligned}
$$

Since $x_{1}$ direction can be chosen arbitrarily, we conclude that $u$ and $v$ must be radially symmetry and monotone decreasing about the origin.

This completes the proof of Theorem 1 .

\section{Appendix}

Here we use the notations in the previous sections. The following theorems are the key ingredients in applying the standard moving planes method which have been explained in [6] with detailed proof. denote

To compare the values of $u(x)$ and $u\left(x^{\lambda}\right)=u_{\lambda}(x)$, we

$$
w_{\lambda}(x)=u_{\lambda}(x)-u(x) .
$$

Theorem A.1 (([6]) (maximum principle for antisymmetric functions)). Let $\Omega$ be a bounded domain in $\Sigma_{\lambda}$. Assume that $w_{\lambda} \in L_{s p} \cap C_{\text {loc }}^{1,1}$ is lower semicontinuous on $\bar{\Omega}$, if

$$
\begin{aligned}
(-\Delta)_{p}^{s} u_{\lambda}(x)-(-\Delta)_{p}^{s} u(x) & \geq 0, \quad x \in \Omega, \\
w_{\lambda}(x) & \geq 0 \quad x \in \Sigma_{\lambda} \backslash \Omega, \\
w_{\lambda}\left(x^{\lambda}\right) & =-w_{\lambda}(x) \quad x \in \Sigma_{\lambda} .
\end{aligned}
$$

Then

$$
w_{\lambda}(x) \geq 0, \quad \forall x \in \Omega .
$$

If $w_{\lambda}(x)=0$ at some point in $\Omega$, then

$$
w_{\lambda}(x)=0 \quad \text { almost everywhere in } \mathbb{R}^{n} .
$$

There conclusions hold for unbounded region $\Omega$ if we further assume that

$$
\varliminf_{|x| \rightarrow \infty} w_{\lambda}(x) \geq 0 .
$$

Theorem A.2 (([6]) (a key boundary estimate)). Assume that $w_{\lambda_{0}}>0$, for $x \in \Sigma_{\lambda_{0}}$. Suppose $\lambda_{k} \longrightarrow \lambda_{0}$, and $x^{k} \in \Sigma_{\lambda_{k}}$, such that

$$
w_{\lambda_{k}}\left(x^{k}\right)=\min _{\Sigma_{\lambda_{k}}} w_{\lambda_{k}} \leq 0, \quad x^{k} \longrightarrow x^{0} \in \partial \Sigma_{\lambda_{0}} .
$$

Let

$$
\delta_{k}=\operatorname{dist}\left(x^{k}, \partial \Sigma_{\lambda_{k}}\right) \equiv\left|\lambda_{k}-x_{1}^{k}\right|
$$

Then

$$
\varlimsup_{\delta_{k} \longrightarrow 0} \frac{1}{\delta_{k}}\left\{(-\Delta)_{p}^{s} u_{\lambda_{k}}\left(x^{k}\right)-(-\Delta)_{p}^{s} u\left(x^{k}\right)\right\}<0 .
$$

\section{Data Availability}

The data used to support the findings of this study are included within the article.

\section{Conflicts of Interest}

The authors declare that they have no conflicts of interest.

\section{Authors' Contributions}

Linfen Cao participated in the method of moving plane studies in the paper; Xiaoshan Wang carried out the evaluation of inequalities and the symmetric theorem; Zhaohui Dai drafted the manuscript. All authors read and approved the final manuscript.

\section{Acknowledgments}

This work is partially supported by HASTIT (no. 15HASTIT012), NSFC (no. 11671121), and the Bureau of Foreign Experts Affairs of Henan Province.

\section{References}

[1] C. Brandle, E. Colorado, A. de Pablo, and U. Sa'nchez, "A concave-convex elliptic problem involving the fractional Laplacian," Proceedings of the Royal Society of Edinburgh, vol. 143, pp. 39-71, 2013.

[2] R. Zhuo, W. Chen, X. Cui, and Z. Yuan, "Symmetry and nonexistence of solutions for a nonlinear system involving the fractional Laplacian," Discrete and Continuous Dynamical Systems Series A, vol. 36, no. 2, pp. 1125-1141, 2016. 
[3] W. Chen, C. Li, and Y. Li, "A direct method of moving planes for the fractional Laplacian," Advances in Mathematics, vol. 308, pp. 404-437, 2017.

[4] W. Chen, C. Li, and G. Li, "Maximum principles for a fully nonlinear fractional order equation and symmetry of solutions," Calculus of Variations and Partial Differential Equations, vol. 56, no. 2, 18 pages, 2017.

[5] Z. Dai, L. Cao, and P. Wang, "Liouville type theorems for the system of fractional nonlinear equations in $\mathrm{R}+\mathrm{n}$," Journal of Inequalities and Applications, vol. 267, 17 pages, 2016.

[6] W. Chen and C. Li, "Maximum principles for the fractional p-Laplacian and symmetry of solutions," Analysis of PDEs, 19 pages, 2017, https://arxiv.org/abs/1705.04891.

[7] L. Caffarelli and L. Silvestre, "Regularity theory for fully nonlinear integro-differential equations," Communications on Pure and Applied Mathematics, vol. 62, no. 5, pp. 597-638, 2009.

[8] P. Wang and P. Niu, "A direct method of moving planes for a fully nonlinear nonlocal system," Communications on Pure and Applied Analysis, vol. 16, no. 5, pp. 1707-1718, 2017.

[9] S. Jarohs and T. Weth, "Symmetry via antisymmetric maximum principles in nonlocal problems of variable order," Annali di Matematica Pura ed Applicata, vol. 195, no. 1, pp. 273-291, 2016.

[10] L. A. Caffarelli and L. Silvestre, "An extension problem related to the fractional Laplacian," Communications in Partial Differential Equations, vol. 32, no. 7-9, pp. 1245-1260, 2007.

[11] L. Cao and Z. Dai, "A Liouville-type theorem for an integral equation on a half-space R+n," Journal of Mathematical Analysis and Applications, vol. 389, no. 2, pp. 1365-1373, 2012. 


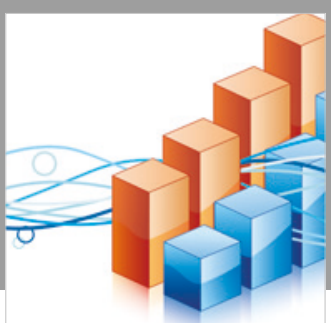

Advances in

Operations Research

\section{-n-m}
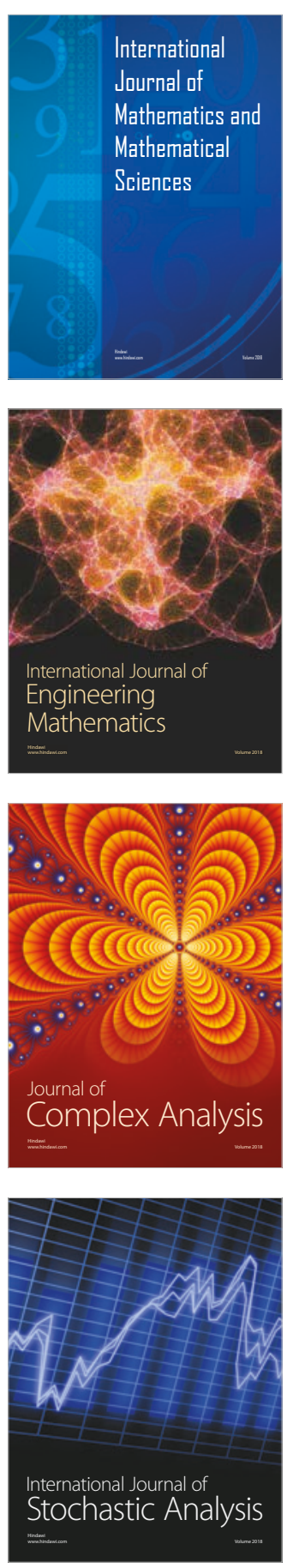
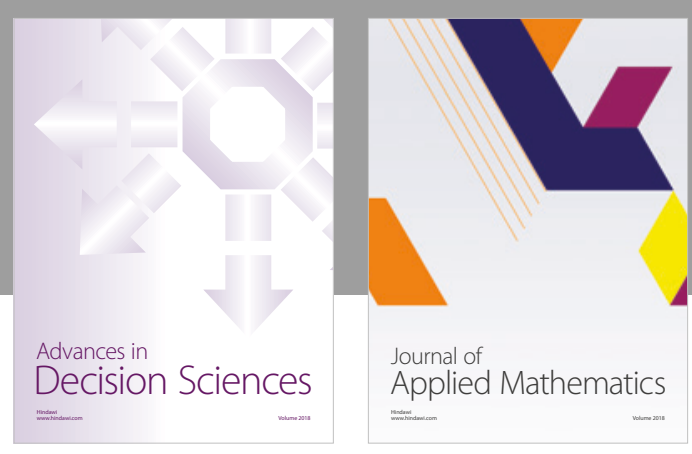

Journal of

Applied Mathematics
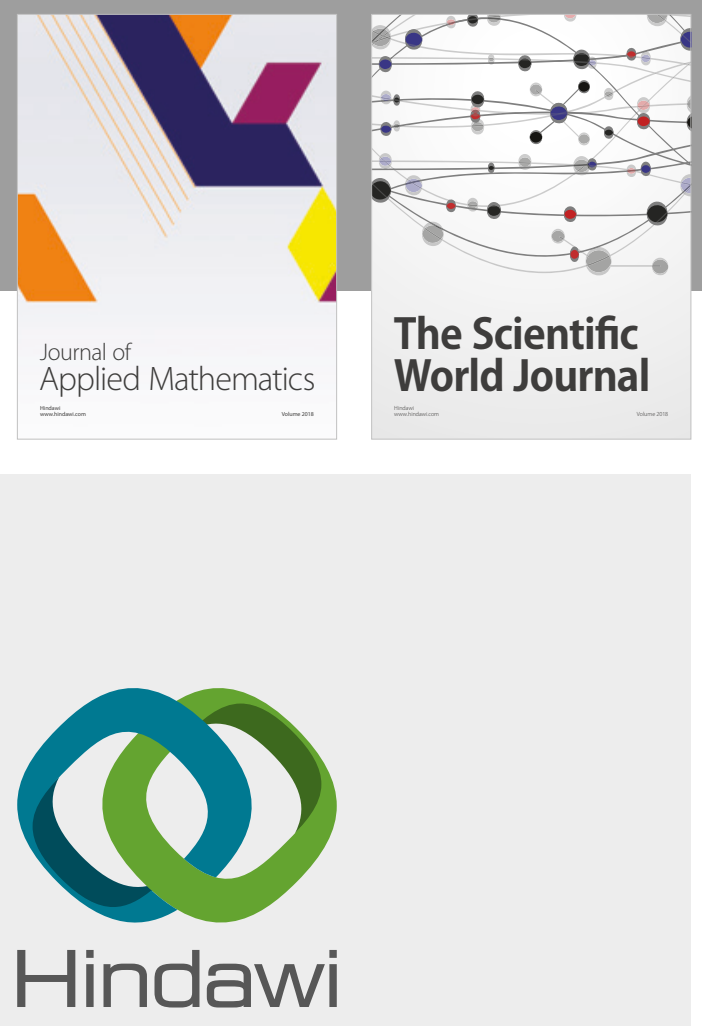

Submit your manuscripts at

www.hindawi.com

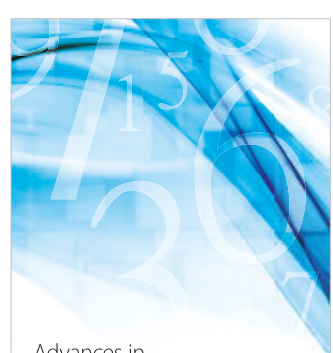

Advances in
Numerical Analysis
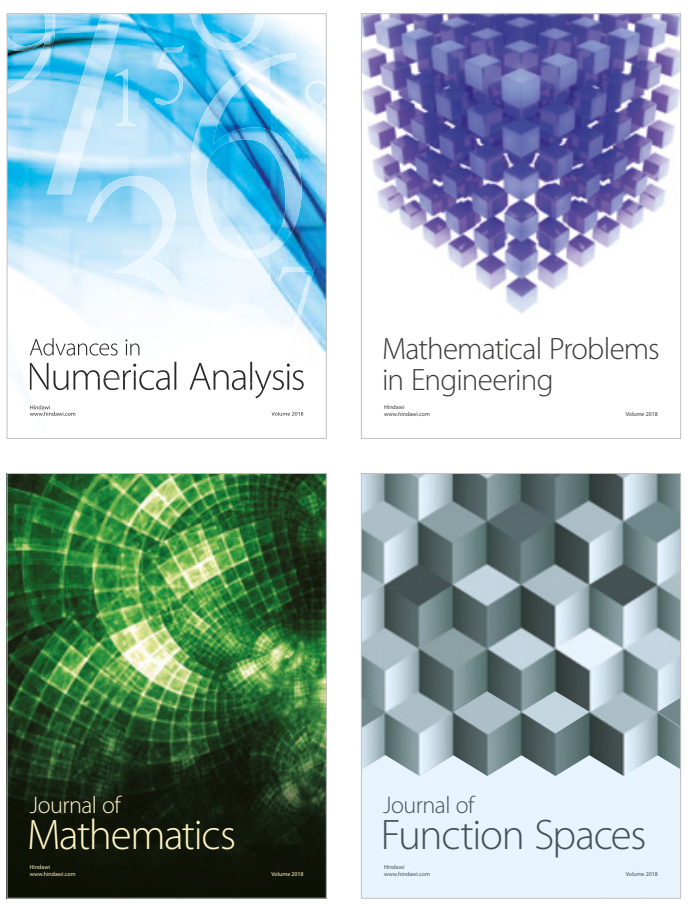

Mathematical Problems in Engineering

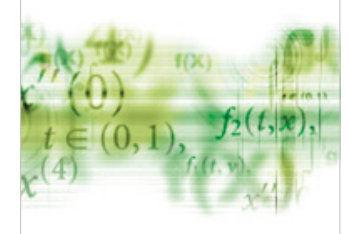

International Journal of

Differential Equations

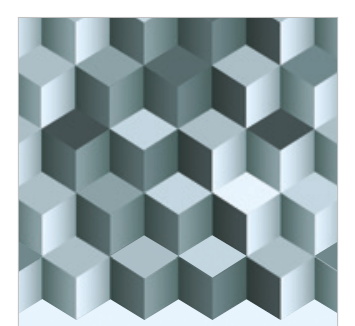

Journal of

Function Spaces

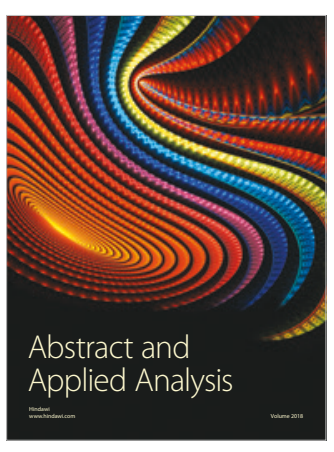

The Scientific

World Journal

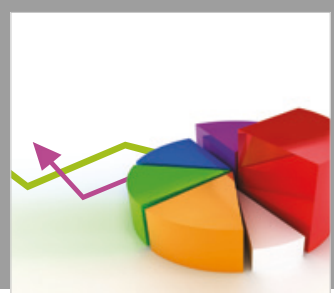

Journal of

Probability and Statistics
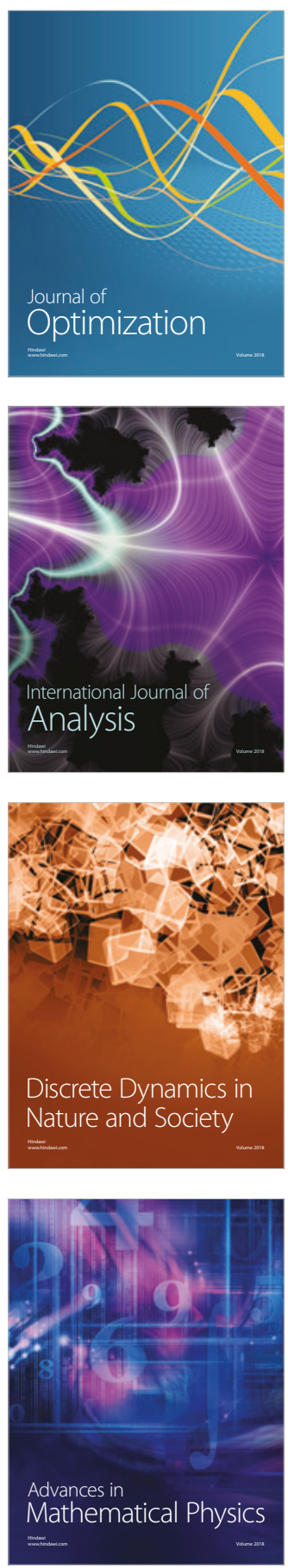